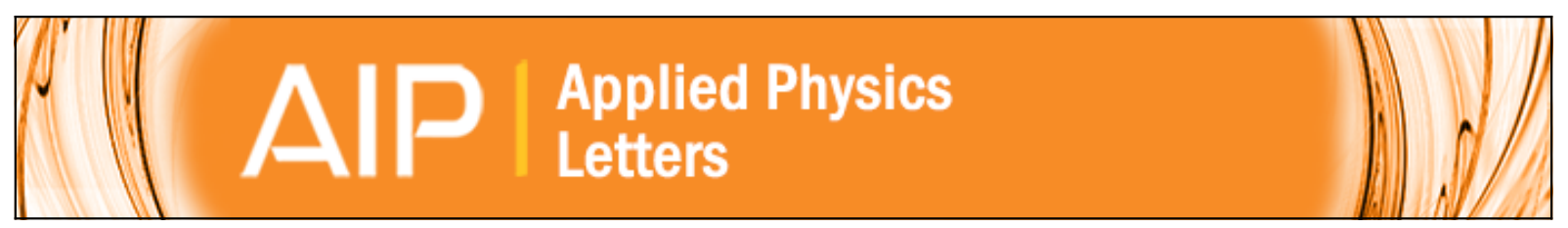

\title{
The surface structure of $\mathrm{SrTiO} 3$ at high temperatures under influence of oxygen
}

M. B. S. Hesselberth, S. J. van der Molen, and J. Aarts

Citation: Applied Physics Letters 104, 051609 (2014); doi: 10.1063/1.4864186

View online: http://dx.doi.org/10.1063/1.4864186

View Table of Contents: http://scitation.aip.org/content/aip/journal/apl/104/5?ver=pdfcov

Published by the AIP Publishing

\section{Articles you may be interested in}

Hydrogen adsorption induced antiferrodistortive distortion and metallization at the (001) surface of SrTiO3

J. Appl. Phys. 118, 105303 (2015); 10.1063/1.4930042

Effect of surface structure on workfunction and Schottky-barrier height in SrRuO3/SrTiO3 (001) heterojunctions J. Appl. Phys. 115, 173705 (2014); 10.1063/1.4872466

Surface electronic structure for various surface preparations of Nb-doped $\mathrm{SrTiO} 3$ (001)

J. Appl. Phys. 114, 103710 (2013); 10.1063/1.4821095

High temperature conductance characteristics of LaAIO 3 / SrTiO 3 -heterostructures under equilibrium oxygen atmospheres

Appl. Phys. Lett. 97, 012103 (2010); 10.1063/1.3457386

Ab initio Calculations for SrTiO3 (100) Surface Structure

AIP Conf. Proc. 626, 285 (2002); 10.1063/1.1499578

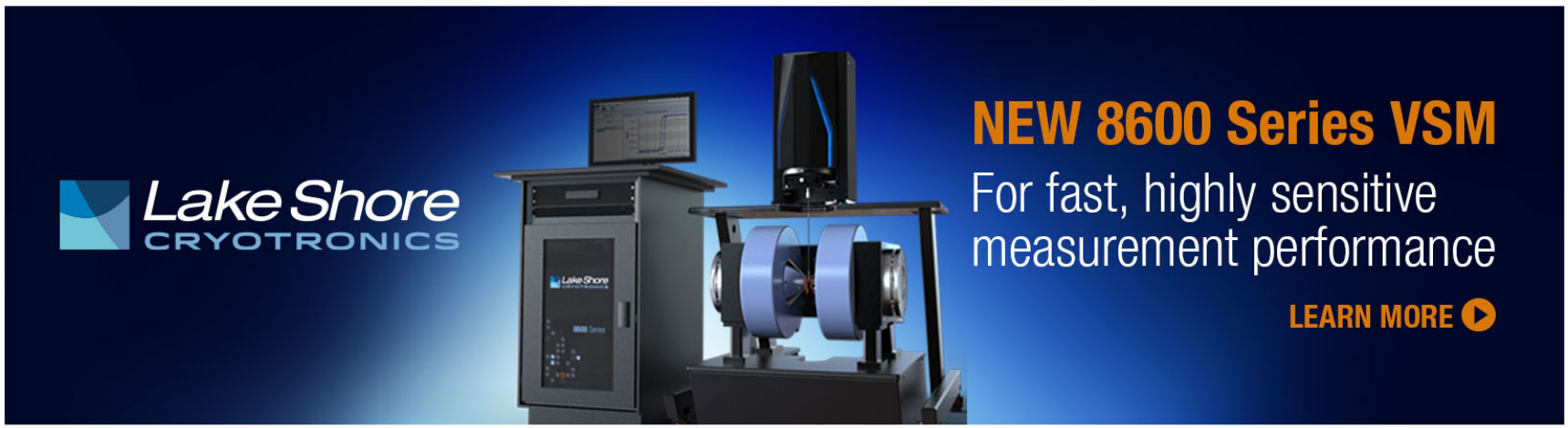




\title{
The surface structure of $\mathrm{SrTiO}_{3}$ at high temperatures under influence of oxygen
}

\author{
M. B. S. Hesselberth, S. J. van der Molen, and J. Aarts \\ Kamerlingh Onnes Laboratory, Leiden University, P.O. Box 9504, 2300 RA Leiden, The Netherlands
}

(Received 28 October 2013; accepted 13 January 2014; published online 7 February 2014)

\begin{abstract}
We use low energy electron microscopy to investigate the structure of the $\mathrm{SrTiO}_{3}(001)$ surface at elevated temperatures and different oxygen pressures. Upon varying the temperature between $500^{\circ} \mathrm{C}$ and $900^{\circ} \mathrm{C}$ in oxygen pressures ranging from $10^{-9}$ millibar to $10^{-4}$ millibar, two surface transitions are found to be present. The lower temperature $(1 \times 1) \rightarrow(2 \times 1)$ transition that is known to occur in ultrahigh vacuum can be reversed by increasing the oxygen pressure. At higher temperatures, we observe a $(2 \times 1) \rightarrow$ disordered $(1 \times 1)$ transition which is irreversible in the experimental parameter range. The observations are expected to have a strong bearing on the growth of interface structures. (C) 2014 AIP Publishing LLC. [http://dx.doi.org/10.1063/1.4864186]
\end{abstract}

In oxide electronics research, the perovskite oxide $\mathrm{SrTiO}_{3}$ (STO) is widely used as substrate material. It is a band insulator with a high dielectric constant, it is relatively inert, and it has a simple square surface net, making it suitable for the growth of a variety of functional oxides. With the advent of atomic-scale growth control, the properties of interfaces have come into the focus of attention. A good example is the realization of a 2-dimensional electron gas at the interface between STO and the band insulator $\mathrm{LaAlO}_{3}$ (LAO). ${ }^{1,2}$ There has been much debate on the mechanisms which lead to the conducting interface, but experimentally important is the fact that it only occurs when the STO surface is singly terminated by a $\mathrm{TiO}_{2}$ layer. ${ }^{1,3}$ The fact that a minimum of 4 unit cells of LAO is required to obtain conductivity ${ }^{4}$ indicates that one of the ingredients is electronic reconstruction (RC), stemming from the charge discontinuity between the neutral $\mathrm{SrO}$ and $\mathrm{TiO}_{2}$ layers versus the singly charged $\mathrm{AlO}_{2}^{-}$and $\mathrm{LaO}^{+}$layers. Growth on the STO surface, mostly performed by Pulsed Laser Deposition (PLD), takes place at temperatures in the range of $700^{\circ} \mathrm{C}-800^{\circ} \mathrm{C}$, and in an oxygen background pressure between $10^{-6}$ millibar and $10^{-2}$ millibar. The resulting interface conductance strongly depends on the oxygen pressure, ${ }^{1,5}$ indicating that the formation of oxygen vacancies plays a role in the conductance as well. This is also found in experiments involving sputter deposition, ${ }^{6}$ or post-annealing. ${ }^{7}$

In view of the influence of the termination layer, the atomic structure of the starting surface is of obvious importance; however, relatively little research has been done to clarify this at actual growth conditions, which entail both a high temperature and a certain oxygen pressure. It is known that a number of surface RCs can be prepared by annealing the crystal at different temperatures and pressures, ranging from $(1 \times 1)$ and $(2 \times 1)$ to $c(4 \times 2),(4 \times 4),(6 \times 2)$, and $(\sqrt{5} \times \sqrt{5}) \mathrm{R} 26.6^{\circ}$, and without full consensus on the exact temperature-pressure phase diagram. A summary of such results can be found in Refs. 8 and 9. The atomic structures of the various RCs are not very well known. ${ }^{10}$ For the $(2 \times 1)$ $\mathrm{RC}$ which is relevant for our experiments, models have been proposed based on removal of oxygen rows, ${ }^{11}$ on reduced surface coverage of $\mathrm{TiO}_{2},{ }^{11}$ or on a double-layer $\mathrm{TiO}_{2}$ termination. ${ }^{12}$ Recent experiments with scanning tunneling microscopy at room temperature (RT) by Phark et al ${ }^{13}$ made the important point that growth of STO or $\mathrm{SrVO}_{3}$ on STO proceeds differently whether a $(2 \times 1)$ or a $\mathrm{c}(6 \times 2) \mathrm{RC}$ has been prepared in advance. However, the authors could not observe the actual starting surface at their deposition condition $\left(600^{\circ} \mathrm{C}-700^{\circ} \mathrm{C}, 10^{-4}\right.$ millibar of oxygen).

Here, we use Low Energy Electron Microscopy (LEEM) to investigate the actual starting surface in a growth process by following the changes in the structure of nominal STO (001) surfaces upon varying the temperature between $500^{\circ} \mathrm{C}$ and $900^{\circ} \mathrm{C}$ in oxygen pressures ranging from $10^{-9}$ millibar to $10^{-4}$ millibar, a range that is relevant for PLD growth. An important finding is that two surface structure transitions are present in this parameter window. The lower temperature $(1 \times 1) \rightarrow(2 \times 1)$ transition that is known to occur in ultrahigh vacuum (UHV) also takes place at low oxygen pressures and can actually be reversed by increasing the oxygen pressure. At higher temperatures, we observe a transition from $(2 \times 1)$ to a disordered $(1 \times 1) \mathrm{RC}$ which we shall call $(1 \times 1)$ DO. This transition we find to be irreversible in the experimentally accessible parameter range.

The instrument used for the experiments is described in Refs. 14-16. It is an aberration corrected low-energy electron microscope (AC-LEEM) that uses a cold field emitter to generate an electron beam with an energy of $15 \mathrm{keV}$. A magnetic prism array bends the electron beam over $90^{\circ}$. The electrons then pass through a cathode objective lens and decelerate to an energy of $0-100 \mathrm{eV}$ at the sample surface. After scattering, they re-accelerate and are bent $90^{\circ}$ by the prism once more so that the illumination beam and image beam are split. The electrons now pass through a second prism array but this time they are reflected by an electrostatic mirror, a procedure which allows corrections for chromatic and spherical aberrations, yielding a lateral image resolution of $<1.4 \mathrm{~nm}$ at optimal conditions. ${ }^{17}$ Projector optics can either magnify the image or can be set to image the back focal plane of the objective lens where the diffraction pattern exists. In the latter mode, microdiffraction can be performed on an area of tens of nm to several $\mu \mathrm{m}$. The base pressure of the instrument is $10^{-10}$ millibar, and the sample can be heated to about $1600^{\circ} \mathrm{C}$ by an electron bombardment heater. 
The temperature of the sample is measured by a pyrometer set to an emissivity of 0.4. A molybdenum plate is placed behind the sample to exclude pyrometer error through light emission of the heater filament. The distance between the sample and the grounded cathode objective lens, where a $15 \mathrm{kV}$ potential difference exists, is of the order of a mm, which allows $\mathrm{O}_{2}$ gas (99.999\% pure) to be admitted to the sample during the experiments. We found that, as expected for the small sample-lens gap of the instrument, $\mathrm{O}_{2}$ pressures up to $10^{-5}$ millibar can be set without risk of creating an arc. Higher $\mathrm{O}_{2}$ pressures can be applied after turning off the sample voltage; in this way, the experimental range can be extended into the $10^{-4}$ millibar range.

The experiments were performed on nominal STO (001) surfaces. Fig. 1(a) shows a typical image of the surface topography made in the LEEM. The field of view is $4 \mu \mathrm{m}$, and the electron energy is $2 \mathrm{eV}$, which yields step contrast through wave interference (phase contrast). Steps can be clearly seen, although they are quite disordered. Next, the sample is heated in the vacuum of the microscope to $900{ }^{\circ} \mathrm{C}$ in a time span of $10 \mathrm{~min}$. The sample can be monitored continuously and images are stored at a rate of 4 frames/s. Two such images are given in Figs. 1(b) and 1(c). The annealing quickly results in the steps straightening out over the full field of view and terrace widths of about $0.3 \mu \mathrm{m}$. Fig. 1(d) presents the surface topography measured by atomic force microscopy (AFM) after heating in the LEEM, showing terraces and unit-cell height steps.

We now analyze the surface structure of STO by selected-area diffraction. To prepare a well defined starting state, the crystals were all pre-treated by BHF etching so that the surface was $\mathrm{TiO}_{2}$-terminated. They were subsequently annealed at $900^{\circ} \mathrm{C}$ in $1 \mathrm{~atm}$ oxygen gas. Figure 2 shows the evolution of the low energy electron diffraction (LEED) pattern upon heating in ultrahigh vacuum. LEED experiments were performed at an electron energy of $38 \mathrm{eV}$. Up to $550^{\circ} \mathrm{C}$, we observe an unreconstructed $(1 \times 1)$ pattern, which changes to a mixture of a $(2 \times 1)$ and a $(1 \times 2)$ pattern between $600^{\circ} \mathrm{C}$
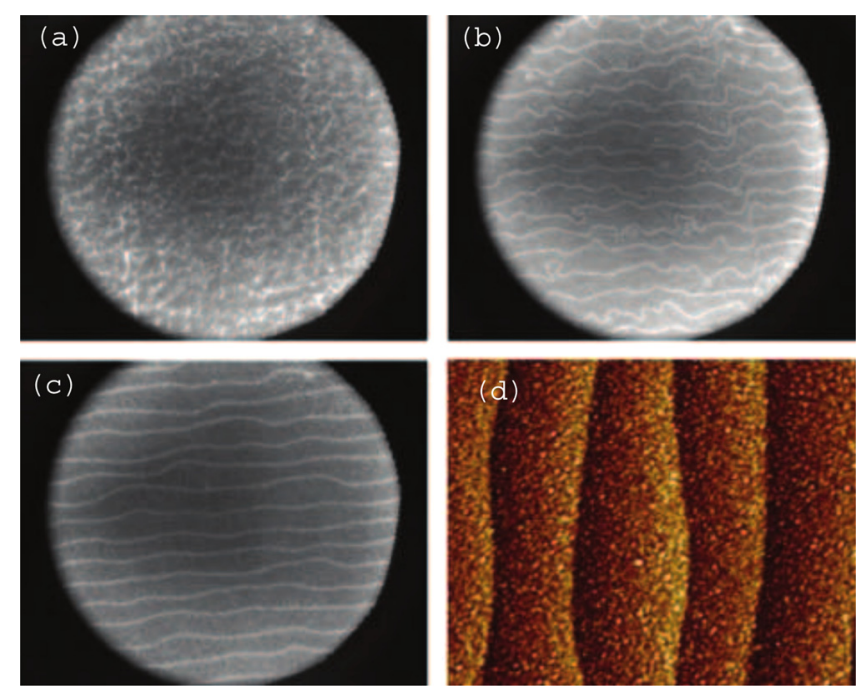

FIG. 1. LEEM images of an STO (001) surface during fast annealing at $900{ }^{\circ} \mathrm{C}$. The field of view is $4 \mu \mathrm{m}$. (a) The initial state; (b) after $5 \mathrm{~min}$; (c) after $10 \mathrm{~min}$ of annealing; and (d) atomic force microscopy image of the same surface obtained after the LEEM experiment. The field of view is $1 \mu \mathrm{m}$. Unit-cell height steps are visible. and $800^{\circ} \mathrm{C}$. The $(2 \times 1) \mathrm{RC}$ sets on at $600^{\circ} \mathrm{C}$ and becomes more developed, showing higher half order peak intensity with increasing temperature. Around a temperature of $850^{\circ} \mathrm{C}$, the $(2 \times 1) \mathrm{RC}$ disappears. The first order spots show decreased intensity, and vestiges of fractional order spots are still present. We designate this state as $(1 \times 1)(\mathrm{DO})$. Above $1000^{\circ} \mathrm{C}$, we see the onset of different fractional order spots which are reminiscent of a $(\sqrt{5} \times \sqrt{5}) \mathrm{R} 26.6^{\circ}$ pattern, although the positions do not match this RC exactly. The large bright spot seen in the LEED patterns at higher temperatures is formed by thermally emitted electrons that are dispersed away from the center of the Ewald sphere by the magnetic prism. The LEED patterns in Fig. 2 form within minutes and are stable up to at least several hours. Comparing these observations to published literature, we note that the $(2 \times 1) \mathrm{RC}$ is ubiquitously reported, but not so a transition to the $1 \times 1$ (DO) RC, although Liang and Bonnell find a $(1 \times 1)$ LEED pattern after similar annealing conditions. ${ }^{18}$ An $(\sqrt{5} \times \sqrt{5}) \mathrm{R} 26.6^{\circ} \mathrm{RC}$, obtained after annealing in UHV at temperatures around $1200^{\circ} \mathrm{C}$, was reported several times, ${ }^{19-21}$ but other RC's can be obtained under various conditions. In particular, the $(\mathrm{c} 6 \times 2) \mathrm{RC}$ is found after annealing in oxygen. ${ }^{13,22-24}$ We observe a $(2 \times 4)$ $\mathrm{RC}$ after long annealing times. Given this variety of possibilities, we believe the $1 \times 1$ (DO) RC should be thought of as a transition regime, where oxygen loss leads to locally different structures or reconstructions.

Since oxygen loss from the surface can be expected to be an important factor in the formation of these RC's, we next study their dependence on oxygen pressure at different temperatures. In these experiments, the temperature was varied between $600^{\circ} \mathrm{C}$ and $850^{\circ} \mathrm{C}$ at three different (constant) values of the oxygen pressure $\left(10^{-5}\right.$ millibar, $10^{-7}$ millibar, UHV). Data were continuously recorded, meaning that the sample high voltage was always present; the maximum pressure of these experiments is therefore limited to $10^{-5}$ millibar. The temperature was kept stable for $>15$ min before acquiring LEED data. Fig. 3 summarizes the results. In $\mathrm{UHV}$, they are the same as shown in Fig. 2: at $550^{\circ} \mathrm{C}$ an unreconstructed surface is observed, at $600^{\circ} \mathrm{C}$ the $(2 \times 1)$ $\mathrm{RC}$ appears, followed by the disordered $(1 \times 1)$ at $850^{\circ} \mathrm{C}$. At $10^{-7}$ millibar, we find essentially the same behaviour. At $10^{-5}$ millibar, the formation of the $(2 \times 1) \mathrm{RC}$ sets on at the slightly higher temperature of $650^{\circ} \mathrm{C}$ and is still stable at $850^{\circ} \mathrm{C}$. Sweeping the oxygen pressure between $10^{-9}$ millibar and $10^{-5}$ millibar does not significantly change the surface at the intermediate temperatures of $650{ }^{\circ} \mathrm{C}$ and $750{ }^{\circ} \mathrm{C}$. At $600^{\circ} \mathrm{C}$, after reducing the pressure below $10^{-5}$ millibar
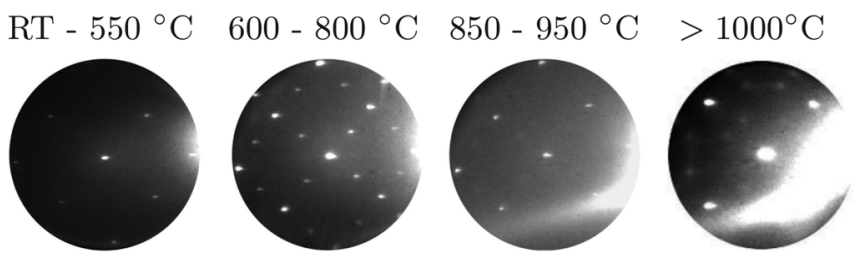

FIG. 2. Images from selected-area diffraction of the STO (001) surface taken at different temperatures as indicated, under ultrahigh vacuum conditions. From left to right (i) between RT and $550{ }^{\circ} \mathrm{C}$ the surface is unreconstructed; (ii) between $600^{\circ} \mathrm{C}$ and $800^{\circ} \mathrm{C}$ a $(2 \times 1) \mathrm{RC}$ develops; (iii) between $850^{\circ} \mathrm{C}$ and $950^{\circ} \mathrm{C}$ a (disordered) $(1 \times 1)(\mathrm{DO}) \mathrm{RC}$ is observed; and (iv) above $1000^{\circ} \mathrm{C}$ new fractional order spots start to develop. 


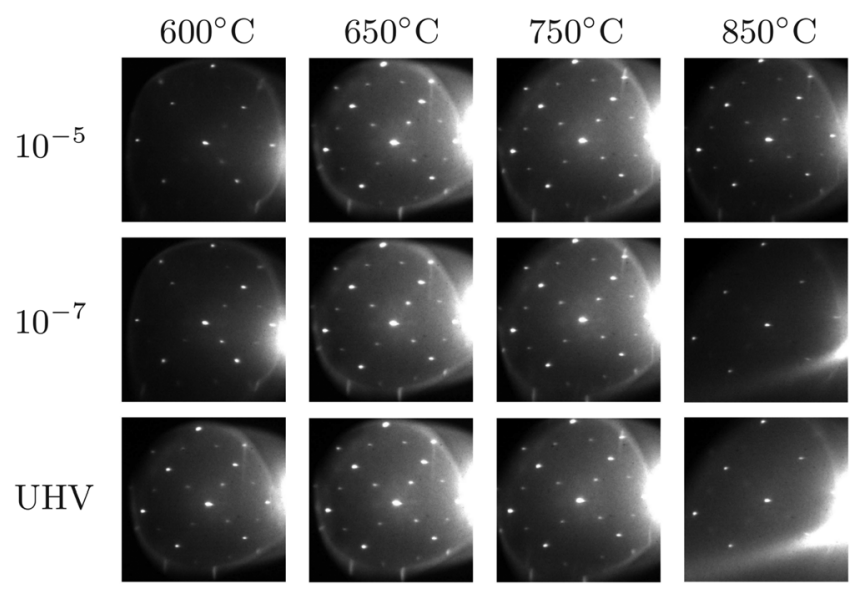

FIG. 3. Selected-area diffraction patterns of the STO (001) surface taken by varying the temperature between $600^{\circ} \mathrm{C}$ and $850^{\circ} \mathrm{C}$ at different constant oxygen pressures. Top: $10^{-5}$ millibar; middle: $10^{-7}$ millibar; bottom: ultrahigh vacuum.

the $(2 \times 1) \mathrm{RC}$ forms and when the pressure is increased to $10^{-5}$ millibar again the $(2 \times 1) \mathrm{RC}$ remains. At $850^{\circ} \mathrm{C}$, the $(2 \times 1) \mathrm{RC}$ is still stable at $10^{-5}$ millibar. When reducing the pressure, the half order spots strongly reduce in intensity and the $(1 \times 1)(\mathrm{DO})$ surface with the decreased specular and first order beam intensities is formed. Increasing the pressure again to $10^{-5}$ millibar does not recover the $(2 \times 1) \mathrm{RC}$.

To conclude, $10^{-5}$ millibar of oxygen (the maximum used in this set of experiments) significantly delays the onset of the surface structure transitions with the $(1 \times 1) \rightarrow(2 \times 1)$ about $50^{\circ} \mathrm{C}$ higher and the $(2 \times 1) \rightarrow(1 \times 1)(\mathrm{DO})$ about $100^{\circ} \mathrm{C}$ higher than in UHV or at $10^{-7}$ millibar. Both transitions are irreversible at this pressure.

To further investigate the role of oxygen the experimental procedure is now changed. At constant temperature, the sample voltage is switched off and the LEEM chamber is filled with an oxygen pressure of $2 \times 10^{-4}$ millibars. After keeping the sample under these conditions for $20 \mathrm{~min}$, the pressure is quickly reduced to about $2 \times 10^{-5}$ millibars, the voltage is turned on and the LEED pattern is recorded immediately. We first concentrate on the transition between the low temperature $(1 \times 1)$ and the $(2 \times 1)$ RC. Fig. 4(a) shows the LEED pattern of the $(2 \times 1)$ surface, as it exists in UHV at $750{ }^{\circ} \mathrm{C}$. After filling with oxygen for $20 \mathrm{~min}$, the structure has reversed to mainly $(1 \times 1)$, as shown in Fig. 4(b). Upon reducing the pressure to the UHV range, the $(2 \times 1) \mathrm{RC}$ reappears as shown in Fig. 4(c). This strongly suggests that (a) UHV

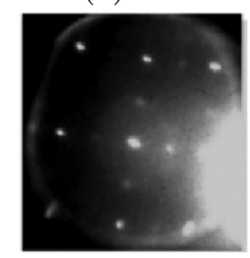

(b) Oxygen

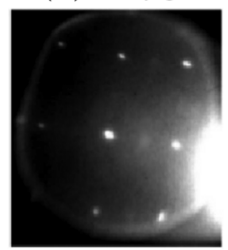

(c) UHV

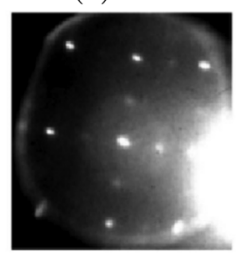

FIG. 4. Selected-area diffraction patterns taken of the STO (001) surface before and after annealing at $750^{\circ} \mathrm{C}$ in $2 \times 10^{-4}$ millibars oxygen. (a) Starting pattern under UHV conditions, with the $(2 \times 1)$ RC present; (b) after increasing the $\mathrm{O}_{2}$ pressure to $2 \times 10^{-4}$ millibars for $20 \mathrm{~min}$ and going down to $2 \times 10^{-5}$ millibars, a strong reduction in the half order spot intensity is observed. (c) After the pressure has been reduced again to UHV, the $(2 \times 1)$ RC reappears.

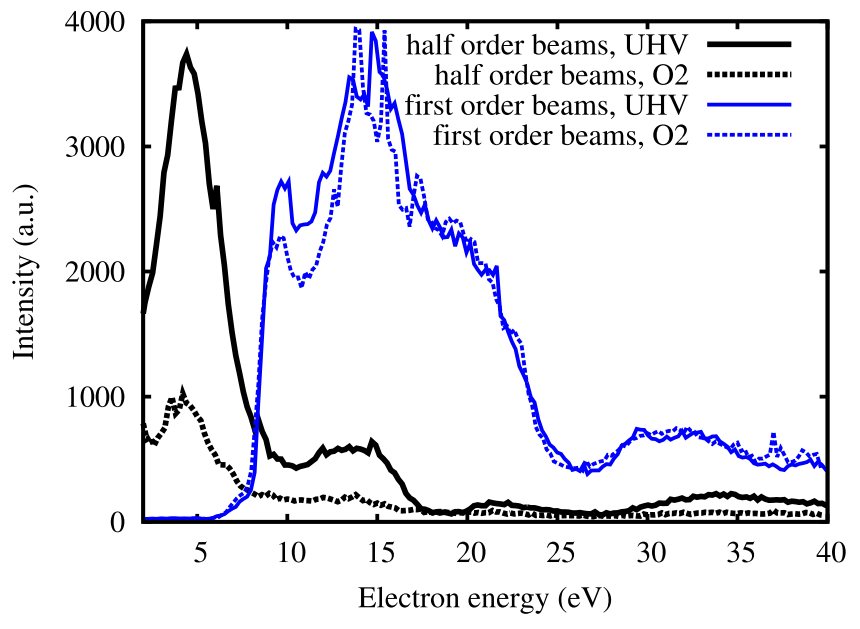

FIG. 5. LEED IV spectra at $750{ }^{\circ} \mathrm{C}$ in UHV before oxygen filling and after annealing in $2 \times 10^{-4}$ millibars of oxygen for $20 \mathrm{~min}$ and pumping back to $2 \times 10^{-5}$ millibars. The half order spot intensity before (after) filling is given by the black line (black dots). The first order spot intensity before (after) filling is given by the blue line (blue dots).

oxygen loss is the driving factor for the formation of the $(2 \times 1) \mathrm{RC}$, and that this can be reversed at a high enough oxygen pressure (in this case, $2 \times 10^{-4}$ millibars).

To quantify this experiment at $750{ }^{\circ} \mathrm{C}$ further, the spot intensity (I) was recorded upon varying the electron energy (V), a so-called IV spectrum. For the specular, half and first order spots, the intensity was computed from the LEED images, using all existing LEED spots of each order and correcting for the secondary electron background. The spot intensity is plotted as a function of energy in Fig. 5. The UHV experiment with the high intensity half order peak at $6 \mathrm{eV}$ was recorded first. Subsequently, the oxygen pressure was increased to $2 \times 10^{-4}$ millibars for $20 \mathrm{~min}$ and reduced again to $2 \times 10^{-5}$ millibars. This reduced the peak in the half order spectrum by a factor 0.26 while shifting it slightly to lower energy. Reversibility was therefore not complete, but that is probably due to the limited annealing time and the short pressure reduction necessary for the analysis.

Performing the same experiment at $650{ }^{\circ} \mathrm{C}$ gives a similar result but a smaller reduction of the $(2 \times 1)$ signal is observed.

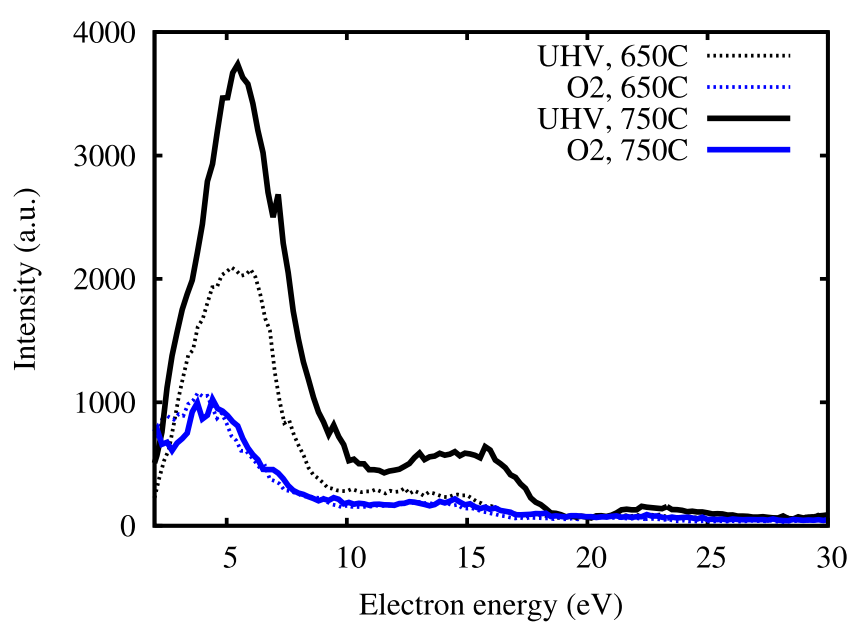

FIG. 6. Comparison of the half order LEED IV spectra at $650{ }^{\circ} \mathrm{C}$ and at $750{ }^{\circ} \mathrm{C}$. The spot intensity at $650{ }^{\circ} \mathrm{C}$ before (after) filling is given by the black (blue) dots. The spot intensity at $750{ }^{\circ} \mathrm{C}$ before (after) filling is given by the black (blue) lines. 
The comparison between the half order IV curves at $650^{\circ} \mathrm{C}$ and at $750{ }^{\circ} \mathrm{C}$ is shown in Fig. 6 . Note that the curves for the two different temperatures are similar after the chamber has been filled with oxygen; the difference is in the initial state, where the $(2 \times 1) \mathrm{RC}$ is seen to be much more complete at $750^{\circ} \mathrm{C}$ than at $650^{\circ} \mathrm{C}$. For the $(1 \times 1) \rightarrow(2 \times 1)$ transition, the experiments clearly show the influence of oxygen yielding a higher transition temperature and at least partial reversibility during the annealing time used. For the higher temperature $(2 \times 1) \rightarrow(1 \times 1)(\mathrm{DO})$, we also find the higher transition temperature in oxygen, but we do not observe reversibility: cooling down from the $(1 \times 1)(\mathrm{DO}) \mathrm{RC}$ does not recover the $(2 \times 1)$ RC. This appears to be a question of the parameter window, since at high temperatures and in atmospheric oxygen pressures, the starting $1 \times 1$ surface can be recovered. In our experimental window this was not possible: the pressure could not be increased further without destabilizing the sample temperature; and samples heated above $900^{\circ} \mathrm{C}$ at low oxygen pressure were observed to become bulk conducting with a sheet resistance in the $\mathrm{k} \Omega$ range.

As a final remark, we note that the observations open a different perspectives onto the issue of the exact structure details of interfaces formed with $\mathrm{SrTiO}_{3}$. There are indications from surface X-ray scattering ${ }^{25,26}$ that the $(2 \times 1)$ reconstruction actually is the double layer $\mathrm{TiO}_{2}$ termination proposed in Ref. 12. More recently, a double layer reconstruction was observed underneath a few layers of $\mathrm{LaMnO}_{3}$ in medium energy ion scattering experiments. ${ }^{27}$ It has been discussed in both cases that the existence of the double layer reconstruction might be connected to the intermixing of the various ions which is often observed. Our experiments emphasize that the temperature window where the reconstruction exists depends on the oxygen pressure, and that interface properties may well depend on the homogeneity and stability of the surface reconstruction at the growth conditions.

We thank R. M. Tromp, A. J. H. van der Torren, and D. B. Boltje and acknowledge financial support from the "NWO-groot" project ESCHER.
${ }^{1}$ O. Ohtomo and H. Hwang, Nature 427, 423 (2004).

${ }^{2}$ D. G. Schlomm and J. Mannhart, Nat. Mat. 10, 168 (2011).

${ }^{3}$ J. Nishimura, A. Ohtomo, A. Ohkubo, Y. Murakami, and M. Kawasaki, Jpn. J. Appl. Phys., Part 2 43, L1032 (2004).

${ }^{4}$ S. Thiel, G. Hammerl, A. Schmehl, C. W. Schneider, and J. Mannhart, Science 313, 1942 (2006).

${ }^{5}$ G. Herranz, M. Basletic, M. Bibes, C. Carretero, E. Tafra, E. Jacquet, K. Bouzehouane, C. Deranlot, A. Hamzic, J.-M. Broto, A. Barthelemy, and A. Fert, Phys. Rev. Lett. 98, 216803 (2007).

${ }^{6}$ I. M. Dildar, D. B. Boltje, M. B. S. Hesselberth, J. Aarts, Q. Xu, H. W. Zandbergen, and S. Harkema, Appl. Phys. Lett. 102, 121601 (2013).

${ }^{7}$ C. Cancellieri, N. Reyren, S. Gariglio, A. D. Caviglia, A. Féte, and J.-M. Triscone, Europhys. Lett. 91, 17004 (2010).

${ }^{8}$ N. Erdman and L. D. Marks, Surf. Sci. 526, 107 (2003).

${ }^{9}$ T. Kubo and H. Nozoye, Surf. Sci. 542, 177 (2003).

${ }^{10}$ K. Johnston, M. R. Castell, A. T. Paxton, and M. W. Finnis, Phys. Rev. B 70, 085415 (2004).

${ }^{11}$ M. R. Castell, Surf. Sci. 505, 1 (2002).

${ }^{12}$ N. Erdman, K. R. Poeppelmeier, M. Asta, O. Warschkow, D. E. Ellis, and L. D. Marks, Nature 419, 55 (2002).

${ }^{13}$ S.-h. Phark, Y. J. Chang, and T. W. Noh, Appl. Phys. Lett. 98, 161908 (2011).

${ }^{14}$ R. M. Tromp, J. B. Hannon, A. W. Ellis, W. Wan, A. Berghaus, and O. Schaff, Ultramicroscopy 110, 852 (2010).

${ }^{15}$ S. M. Schramm, J. Kautz, A. Berghaus, O. Schaff, R. M. Tromp, and S. J. van der Molen, IBM J. Res. Dev. 55, 1.1 (2011).

${ }^{16}$ R. M. Tromp, J. B. Hannon, W. Wan, A. Berghaus, and O. Schaff, Ultramicroscopy 127, 25 (2013).

${ }^{17}$ S. M. Schramm, S. J. van der Molen, and R. M. Tromp, Phys. Rev. Lett. 109, 163901 (2012).

${ }^{18}$ Y. Liang and D. A. Bonnell, Surf. Sci. 285, L510 (1993).

${ }^{19}$ H. Tanaka, T. Matsumoto, T. Kawai, and S. Kawai, Jpn. J. Appl. Phys., Part 1 32, 1405 (1993).

${ }^{20}$ R. Akiyama, T. Matsumoto, H. Tanaka, and T. Kawai, Jpn. J. Appl. Phys., Part 1 36, 3881 (1997).

${ }^{21}$ T. Kubo and H. Nozoye, Phys. Rev. Lett. 86, 1801 (2001).

${ }^{22}$ M. Naito and H. Sato, Physica C 229, 1 (1994).

${ }^{23}$ Q. Jiang and J. Zehenhagen, Surf. Sci. 367, L42 (1996).

${ }^{24}$ C. H. Lanier, A. van de Walle, N. Erdman, E. Landree, O. Warschkow, A. Kazimirov, K. R. Poeppelmeier, J. Zegenhagen, M. Asta, and L. D. Marks, Phys. Rev. B 76, 045421 (2007).

${ }^{25}$ P. R. Willmott, S. A. Pauli, R. Herger, C. M. Schlepütz, D. Martoccia, B. D. Patterson, B. Delley, R. Clarke, D. Kumah, C. Cionca, and Y. Yacoby, Phys. Rev. Lett. 99, 155502 (2007).

${ }^{26}$ R. Herger, P. R. Willmott, O. Bunk, C. M. Schlepütz, B. D. Patterson, B. Delley, V. L. Shneerson, P. F. Lyman, and D. K. Saldin, Phys. Rev. B 76, 195435 (2007).

${ }^{27}$ Yu. A. Boikov, I. T. Serenkov, V. I. Sakharov, A. S. Kalabukhov, P. P. Aurino, D. Winkler, and T. Claeson, EuroPhys. Lett. 102, 56003 (2013). 